\title{
TWO DECADES OF TRANSESOPHAgeal PHASED ARRAY PROBES
}

\author{
K. K. Djoa ${ }^{\dagger}$ N. De Jong, ${ }^{\ddagger}$ A. H. Cromme-Dijkhuis, ${ }^{*}$ \\ C. T. LANCÉE ${ }^{\dagger}$ and N. BOM ${ }^{\ddagger}$ \\ Thoraxcentre Laboratory for Experimental Echocardiography, Erasmus University Rotterdam, \\ The Netherlands; Thoraxcentre Laboratory for Experimental Echocardiography, Erasmus University Rotterdam \\ and the Interuniversity Cardiology Institute of The Netherlands, Rotterdam, The Netherlands; \\ and *Sophia Children's Hospital, University Hospital, Rotterdam, The Netherlands \\ (Received 5 January 1995; in final form 17 May 1995)

\begin{abstract}
After its introduction about two decades ago, transesophageal echocardiography (TEE) has rapidly evolved into an important diagnostic feature for the cardiologist, since it offers anatomic and hemodynamic information which cannot be obtained precordially. Part of this success was due to the developments in transducer technology which resulted in smaller probes with progressively better imaging qualities. A short review of past, recent and future developments of TEE phased array probes, in particular those at the Erasmus University in Rotterdam, will be given. Furthermore, this article discusses basic parameters of the transducer dictating image quality such as centre frequency, array aperture and focusing illustrated with several simulations. The simulations show that a poor design of the transducer will limit the resolution and will give artefacts in the two-dimensional image.
\end{abstract}

Key Words: Ultrasound, Echocardiology, Transesophageal, Transducers.

\section{INTRODUCTION}

The history of ultrasound in cardiology started in 1954 when Edler and Hertz used a single element transducer to produce an A-mode image on a normal cathode ray tube (Edler and Hertz 1954). This important breakthrough initiated the development of M-mode echocardiography. In the period from 1960 until early 1970 great progress was being made in real-time two-dimensional (2D) imaging through advances in transducer technology, electronics and theoretical acoustic fundamentals. True real-time 2D imaging became possible when Bom (1972) constructed a linear array scanner.

However, the rather large footprint of this linear array device limited the applicability for transthoracic echocardiography (TTE), and stimulated the development of sector scanners in cardiology. In sector scanners a 2D sector image is produced by an acoustic beam originating from a single point. Compared to lincar scanners, the contact area of sector scanners is therefore smaller, making it more suitable not only for transthoracic

Address correspondence to: K. K. Djoa, Thoraxcentre Ee 2302, Erasmus University Rotterdam, P.O. Box 1738, NL-3000 DR Rotterdam. The Netherlands. "in between the ribs" application, but especially for transesophageal echocardiography (TEE).

Just as in transthoracic ultrasound, in current TEE sector scanners the ultrasound beam can be pointed either mechanically or electronically in different directions needed to produce a cross-sectional image. In mechanical sector scanners the transducer is mechanically pointed in the desired directions. In electronic sector scanners, better known as phased arrays, the transducer is fixed in position while the beam is steered electronically by modifying the phase relationships of individual linear transducer elements. In this study only phased array TEE sector scanners will be discussed.

The first cardiac ultrasound investigation from the esophagus involved continuous-wave Doppler recordings of cardiac flow reported by Side and Gosling (1971). In 1976 the fundamentals of M-mode diagnosis from within the esophagus were established by Frazin et al. (1976). Imaging from the esophagus began in 1977 when Hisanaga and coworkers reported a 2D real-time scanning system, consisting of a rotating single element in a liquid-filled balloon mounted at the tip of a gastroscope (Ilisanaga et al. 1977). One year later they also described a mechanical linear scanning device for transesophageal use (Hisanaga et al. 1978). 
The next important stage in the development of TEE transducers was the introduction of electronic sector scanners. In 1980, DiMagno and colleagues described a high-frequency (10-MHz) linear array for scanning of small parts (DiMagno et al. 1980). Two years later, Souquet and Hanrath (Souquet et al. $1982 \mathrm{a}$ ) introduced the first transesophageal 3.5-MHz electronic sector scanner based on the phased array principle previously described by Somer (1968) and Thurstone et al. (1974). This 32-element electronic sector scanner was connected to a commercial Varian V3400 ultrasound unit. From this moment on phased array scanning from the esophagus experienced a rapid evolution.

At that time images were of mediocre quality while the size of the probes was quite large. The Laboratory for Experimental Echocardiography of the Erasmus University in Rotterdam developed a series of transducers with progressively better image quality and decreasing probe scanhead dimensions. In our institute one of the first constructed transesophageal phased array transducers was reported in 1982 (Lancée et al. 1982). This design featured a 24-element 3.1-MHz transducer with a pitch (centre-to-centre distance of adjacent elements) of $400 \mu \mathrm{m}$. In the following years, advancements in microminiature cutting, bonding technology and selection of lens material resulted in a series of transducers with improved image quality (De Jong et al. 1986).

A design considered optimal for routine clinical application realized in 1985 , was a 64-element 5.6$\mathrm{MHz}$ array having a pitch of only $160 \mu \mathrm{m}$. This design had a very low artefact level (grating lobes) combined with superior lateral and axial resolution (Gussenhoven et al. 1987; Lancée et al. 1988) and soon became a standard configuration for commercially available adult TEE probes. The next section will illustrate these improvements of image resolution.

For application in pediatric cardiology, on the other hand, the size of such standard adult transesophageal transducers and gastroscopes was still a major limitation. Even though TTE in the smaller patient often leads to adequate diagnostic imaging, TEE is mandatory to obtain better delineation of abnormalities found by TTE or when TTE is inadequate because of chest wall abnormalities, lung disease or artificial ventilation. This stimulated institutes and companies around the world to continue the design of advanced, small-sized TEE probes. In Japan two pediatric single plane TEE probes (one longitudinally and the other transversely scanning) were developed by the Aloka Company. Our institute designed a 48-element pediatric single-plane TEE probe in 1989 , which was com- mercialized by Oldelft BV (Delft, The Netherlands) shortly thereafter.

Subsequent developments have included the development of multiplanes (bi- and varioplanes) to overcome the lack of versatility associated with imaging of structures in only one single scan plane (Roelandt et al. 1992a). In addition, efforts have been directed toward the further miniaturisation of phased array transducers and the provision of higher frequencies for improved resolution so that probes can be used in very small children and newborns or perhaps for monitoring of patients in the intensive care unit. Examples are the pediatric biplane and the unique matrix biplane (adult as well as pediatric) probes developed by Aloka (Omoto et al. 1991). In 1993 a very small 7.5-MHz neonatal TEE probe was developed by our institute in close cooperation with Oldelft. In that same year we also realized a pediatric varioplane prototype. Future progress is focused on designing a pediatric high frequency $(7.5-\mathrm{MHz})$ varioplane TEE probe, a combination of the two previously described probes.

\section{IMAGE RESOLUTION}

Image quality depends on the resolution in three dimensions. In ultrasound systems it refers to the ability to distinguish small structures with clarity and is determined by the axial, lateral and transverse transducer response.

\section{Axial resolution}

Axial resolution refers to the ability of the system to separate objects located along the propagation axis of the ultrasound beam. It is determined by the length of the transmitted pulse which is inversely proportional to the frequency. Consequently, higher frequency transducers produce shorter pulses resulting in an improved axial resolution. This is shown in Fig. 1 comparing typical time responses of 3.1-, 5.0- and 7.5$\mathrm{MHz}$ systems.

On the other hand, higher frequency results in an increase of attenuation of ultrasound in tissue. Therefore, efforts to improve resolution by increasing the frequency will decrease the maximum penetration depth. Figure 2 shows the inverse relationship between frequency and scan depth. The shaded area denotes the region where the beam is optimally formed. Typically, this ranges from a half to two times the axial distance of the near-far field transition of the transducer used. In effect, it means a compromise should be obtained betwecn the desired scan depth and axial resolution.

\section{Lateral and transverse resolution}

Lateral resolution refers to the resolution in the scan plane, whereas transverse resolution involves the 


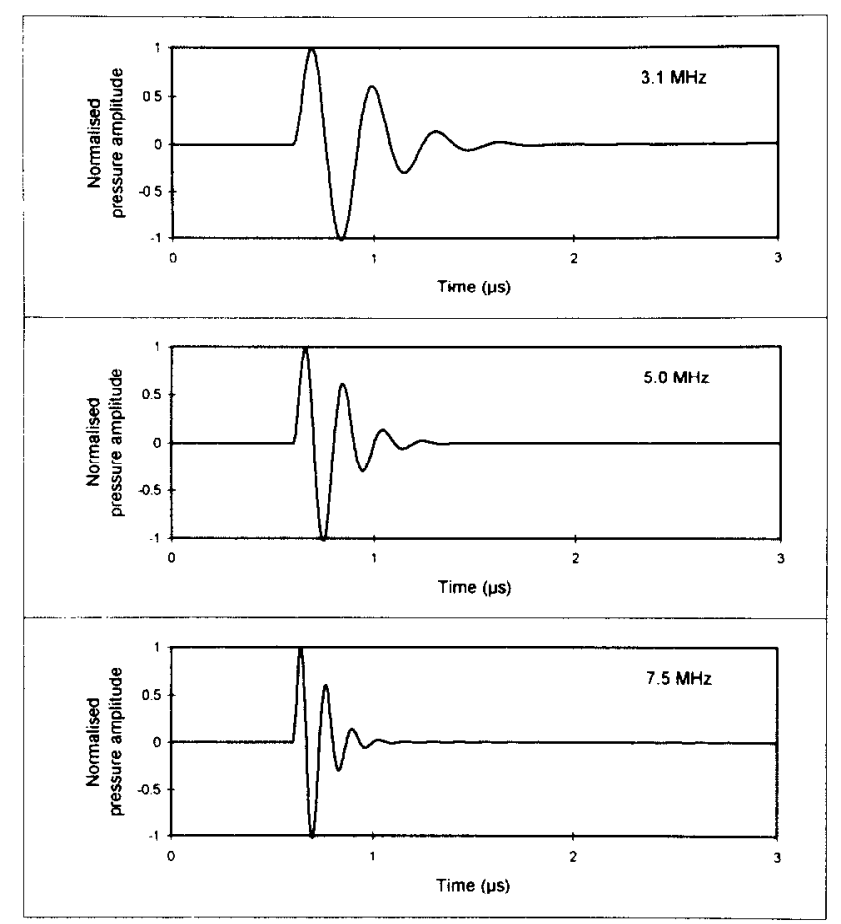

Fig. 1. Typical time responses of 3.1-, 5.0- and 7.5-MHz systems.

resolution perpendicular to the scan plane. Both are determined by the shape of the sound beam which, in general, is characterized by the aperture (determined by number of elements and pitch), the transmitting frequency and by focusing of the transducer. With phased arrays the beam in the scan plane is focused electronically, whereas for transverse focusing a fixed acoustic lens is usually used.
Figure 3 shows the simulated lateral beam profiles of a 3.1- and 5.0-MHz transducer, respectively, representing the first TEE probe prototype used in pigs at the Thorax Centre in 1982 and a typical currently commercially available adult TEE probe. For calculation of the beam profiles the artificial (gamma) time pulse function

$$
f(t)=t e^{-(t / T)}
$$

in which $t$ denotes time and $\tau=4 \mathrm{~s}$ has been used (see also Fig. 1). At each axial distance the acoustic pressure is calculated from $-90^{\circ}$ to $+90^{\circ}$ for a beam steering at $40^{\circ}$. Normalization to the maximum value at each axial distance is carried out to eliminate the axial distance dependency of the acoustic pressure. It can be seen that higher frequencies (with approximately constant aperture) result in narrower beam widths, giving an improved lateral resolution. It should, however, again be noted that increasing the frequency limits the scan depth.

Another important item in lateral resolution is the presence of secondary ultrasound beams (side lobes) outside the main beam (main lobe). In a similar way to that known from grids or gratings in optics, secondary beams are in fact interference patterns generated by the regular element pattern of the arrays, thereby increasing the effective beam width and decreasing the lateral resolution. Side lobes close to the main lobe are caused by the total aperture of the array. In 2D echocardiography, the level of side lobes is minimized by aperture apodization. A cosine weight aperture apodization, for example, can decrease the theoretical side lobe level from $-17 \mathrm{~dB}$ to $23.5 \mathrm{~dB}$.

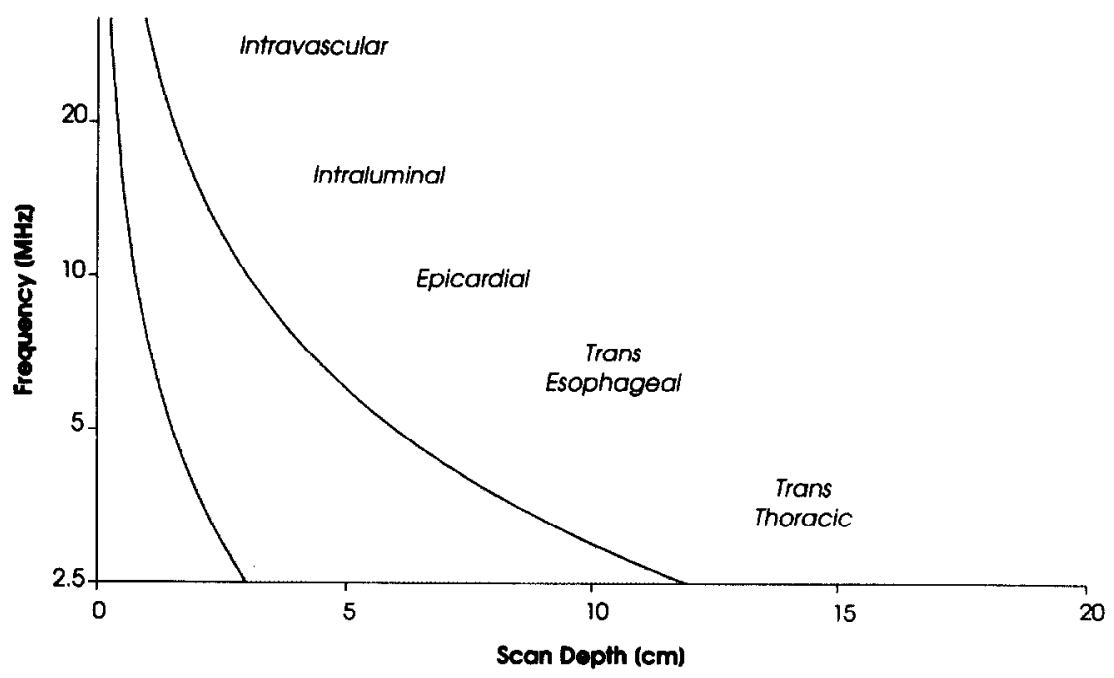

Fig. 2. Inverse relationship between frequency and scan depth. At a given frequency, the shaded area indicates the region from a half to two times the axial distance of the near-far field transition where typically the beam is optimally formed. 
A special side lobe is known as the grating lobe which will appear when the main beam is steered at large angles. Now echoes from the grating lobe direction will be presented in the main beam direction and may therefore be erroneously interpreted as coming from the steering ( = main) beam direction, causing artefacts in the image.

One of the factors determining the appearance of grating lobes is the pitch, $p$, expressing the centre-tocentre distance between adjacent elements. Theoretically, the absence of grating lobes requires the following condition for the pitch, $p$ (Lancée 1987):

$$
p \leq 0.6 \lambda_{\text {min }}
$$

Here, $\lambda_{\min }$ is the wavelength of the highest frequency component in the pulse spectrum.

However, in the practical realization of transducers the pitch is also dictated by the state-of-the-art cutting technology level of the piezoelectric elements, which initially was quite low. But, over the years improvements in this microcutting technology resulted in transducers in which the pitch could be better matched to the above condition. This result can be seen in Fig. 3 illustrating the considerable suppression of grating lobes at about $-50^{\circ}$ in the $5.0-\mathrm{MHz}$ probe.

\section{LATEST TEE PROBE DEVELOPMENTS AT THE ERASMUS UNIVERSITY}

Pediatric high-frequency (7.5-MHz) single-plane TEE probe

An experimental pediatric 48-element $5-\mathrm{MHz}$ TEE probe having a scanhead size of $26 \times 10 \times 5$ mm (length $\times$ width $\times$ thickness) and a shaft diameter of $7 \mathrm{~mm}$ was realized at our institute in 1989. With this probe only children above about $3.5 \mathrm{~kg}$ can be examined, whereas for newborns size is still a restriction. To overcome this problem we developed a very small (but still 48 elements) neonatal TEE probe in close cooperation with Oldelft. Its scanhead dimensions measure $17.5 \times 7.1 \times 5.0 \mathrm{~mm}$, and the shaft diameter is $5 \mathrm{~mm}$. This probe, constructed in 1993. has been successfully used in infants with a weight range from 1.8 to $9 \mathrm{~kg}$ at the Sophia Children's Hospital in Rotterdam. In Fig. 4 this high-frequency (HF) pediatric probe (probe 2 ) is shown together with the pediatric varioplane probe (probe 1) discussed in the next section.

The size reduction was possible due to the increase of the centre frequency from 5.0 to $7.5 \mathrm{MHz}$ and the availability of smaller multiwire electric coaxial cables (AWG 42,63 $\mu \mathrm{m}$ in diameter) and improved backing materials. Apart from size-reducing considerations, using a higher frequency also led to an improved axial resolution. The lateral resolution of the $7.5-\mathrm{MHz}$ transducer, however, is similar to that of a $5.0-\mathrm{MHz}$ transducer since the $7.5-\mathrm{MHz}$ transducer has a smaller aperture so the wavelength-aperture ratio between these two transducers is equivalent. This is shown in the lateral beam profile simulations of Fig. 5 .

The probe has been tested for electrical safety according to international standards (IEC 601-1). In this prototype no thermistor has been included for temperature control. Acoustic measurements reveal a cen-

$\begin{array}{lll}\text { frequency } & 3.1 & \mathrm{MHz} \\ \text { aperture } & 9.6 & \mathrm{~mm} \\ \text { elements } & 24 & \\ \text { pitch } & 0.4 & \mathrm{~mm} \\ \text { focus } & 70 & \mathrm{~mm}\end{array}$

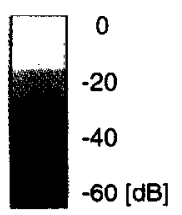

frequency $5.0 \mathrm{MHz}$ aperture $10.2 \mathrm{~mm}$ elements 64 pitch $0.16 \mathrm{~mm}$ focus $70 \mathrm{~mm}$

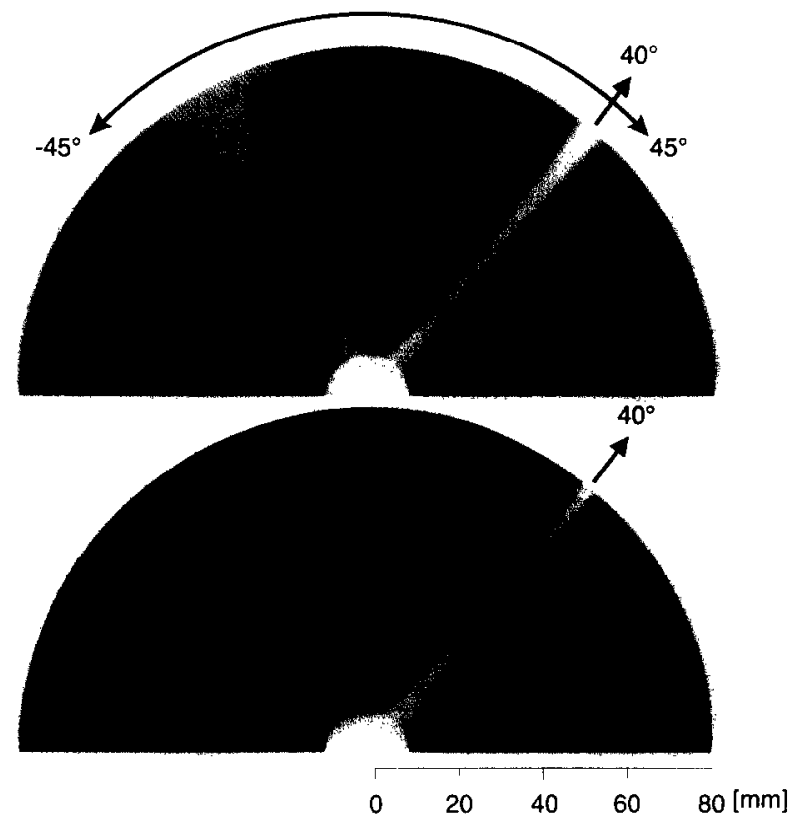

Fig. 3. Simulated lateral beam profiles of a 3.1-MHz (first TEE probe prototype used in pigs at the Thorax Centre in 1982) and a 5.0-MHz (typical commercially available TEE probe configuration) transducer. 


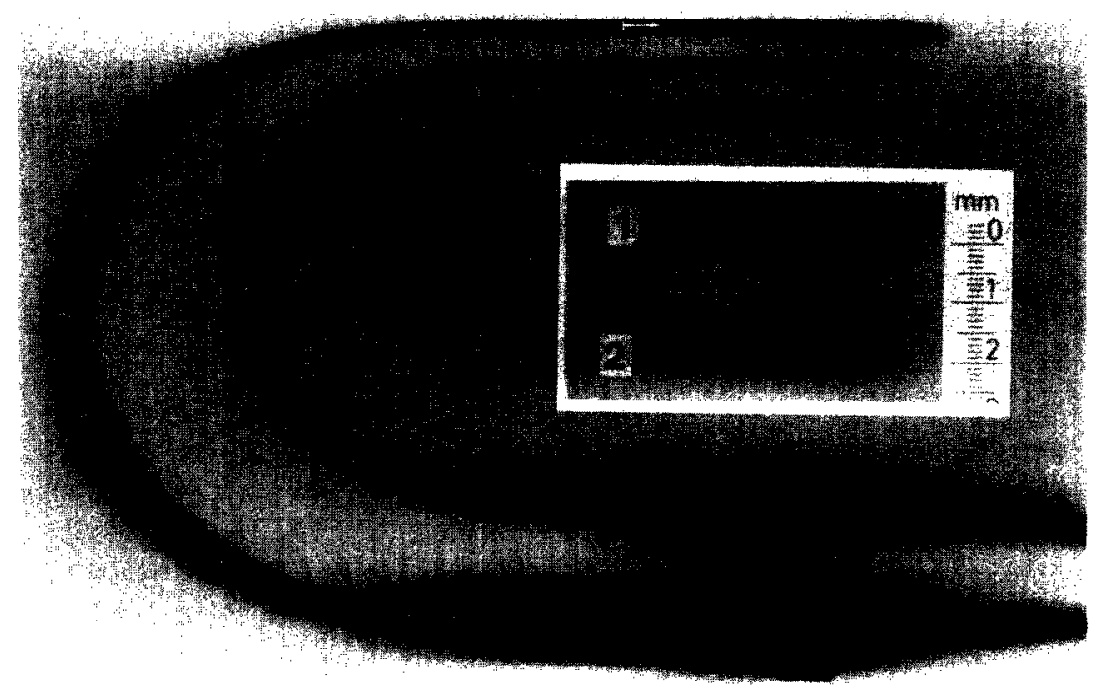

Fig. 4. Photograph of the pediatric high-frequency ( 7.5-MHz) single-plane TEE probe prototype (probe 2: scanhead dimensions [ length $\times$ width $\times$ thickness] : $17.5 \times 7.1 \times 5.0 \mathrm{~mm}$; shaft diameter $5 \mathrm{~mm}$ ) and the pediatric varioplane TEE probe prototype (probe 1: scanhead dimensions [ length $\times$ width $\times$ thickness] : $27.0 \times 10.6 \times 7.9 \mathrm{~mm}$; shaft diameter $7 \mathrm{~mm}$ ).

tre frequency of $6.5 \mathrm{MHz}$, which is probably caused by a too-thin matching layer. The $-20-\mathrm{dB}$ bandwidth is $75 \%$ and the round trip insertion loss $3.0 \mathrm{~dB}$.

Since a higher frequency is related to a higher attenuation, the penetration depth will decrease. However, in infants, only a limited penetration depth is required. The quality of $2 \mathrm{D}$ as well as Doppler imaging (CW, PW, color Doppler) is excellent and comparable to that of standard 5-MHz pediatric TEE probes. The prototype neonatal probe shows nicely detailed struc- tures of the heart especially in the near field. This is illustrated in Fig. 6 showing a ventricular septal defect in a $1.8-\mathrm{kg}$ infant.

\section{Pediatric ( $5-\mathrm{MHz}$ ) varioplane TEE probe}

The idea of multiplane scanning was launched as early as 1982 by Souquet and Hanrath (Hanrath et al. 1982; Souquet 1982) who reported on biplane scanning to deal with the lack of versatility related to single-plane scanning. Clearly, even more flexibility can
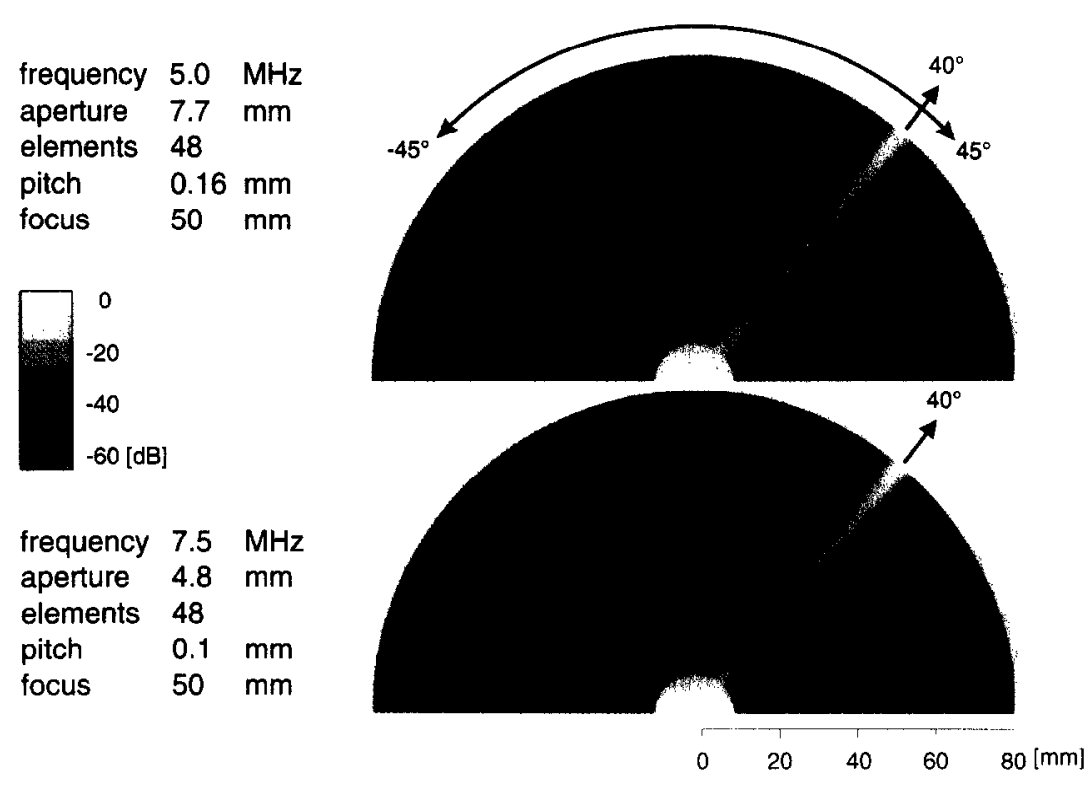

Fig. 5. Simulated lateral bcam profiles of a $5.0-\mathrm{MHz}$ and a $7.5-\mathrm{MHz}$ pediatric 48 -element transducer. 
be gained if all planes between the transverse and longitudinal planes are accessible, for example, by rotating the transducer array. The realization of such a vario- or omniplane transducer, also suggested by Souquet and Hanrath ( 1982), is accompanied by several technical problems.

These problems includes the connection of the array, the rotation mechanism, the sealing and the array shape. In 1991, our institute constructed a prototype adult varioplane TEE probe which has been used with success in over 160 patients (Roelandt et al. 1992b). Also at that time the design of a pediatric version of the varioplane was initiated and, at the end of 1993 , the first prototype pediatric varioplane became available for clinical evaluation.

Figure 4 shows a photograph of the pediatric varioplane (probe 1). The scanhead dimensions of this 5$\mathrm{MHz} 48$-element TEE probe measure $27 \times 10.6 \times 7.9$ mm (length $\times$ width $\times$ thickness) and the shaft diameter $7 \mathrm{~mm}$. Unlike the adult varioplane, the pediatric version rotates internally so there are no moving (rotating) elements in contact with the esophagus. On the photograph the circular acoustic window on the scanhead beneath which the ceramic is located can be seen clearly. The schematic cutaway drawings of Fig. 7 illustrate some of the construction details.

Between the window and ceramic there is a gap of about $50 \mu \mathrm{m}$ which is filled with an acoustic coupling fluid. The circular-shaped ceramic is housed in a stainless-steel cylinder and fits in a circular cavity in the scanhead which acts as a bearing for the cylinder and as a seal for the acoustic coupling fluid. The bottom of the cylinder filled with backing material is connected to a pulley by two pins. By pulling a Bowden cable attached to the pulley, the pulley, and therefore the cylinder with ceramic, can be rotated.

Two Kapton flexible prints with 24 copper leads on each print are attached along the circumference of the ceramic with conductive adhesive. A third ground flex is attached along the other side of the ceramic. The ceramic is then diced into 48 elements so that a one-to-one connection is created between the elements and the copper leads. Each copper lead in turn is connected to a AWG 42 coaxial cable. The 48 coaxial cables are bundled in two groups of 24 coaxial leads. The hraidings of 24 coaxial leads in each group are tied together, soldered to the ground flex and used as the returning ground for the two groups of 24 elements.

The ceramic is backed with a mix of silicone rubber and Wolfram powder which ensures an acoustic attenuation of about $30 \mathrm{~dB} / \mathrm{mm}$ at $5 \mathrm{MHz}$. A mix of Araldite epoxy and Silicon carbide powder with an acoustic impedance $Z \approx 6.5$ MRayl is used to match the ceramic to the load. This matching layer also acts as a concave acoustic lens (radius, $R=50 \mathrm{~mm}$ ). RTV rubber is used to "flatfill" the concave matching layer to a plane top layer. This is necessary because an nonflat top layer may induce the creation of air bubbles in the acoustic coupling fluid between the top layer and the window during rotation.

Like the prototype neonatal $7.5-\mathrm{MHz}$ frequency probe, the pediatric varioplane meets the IEC 601-1

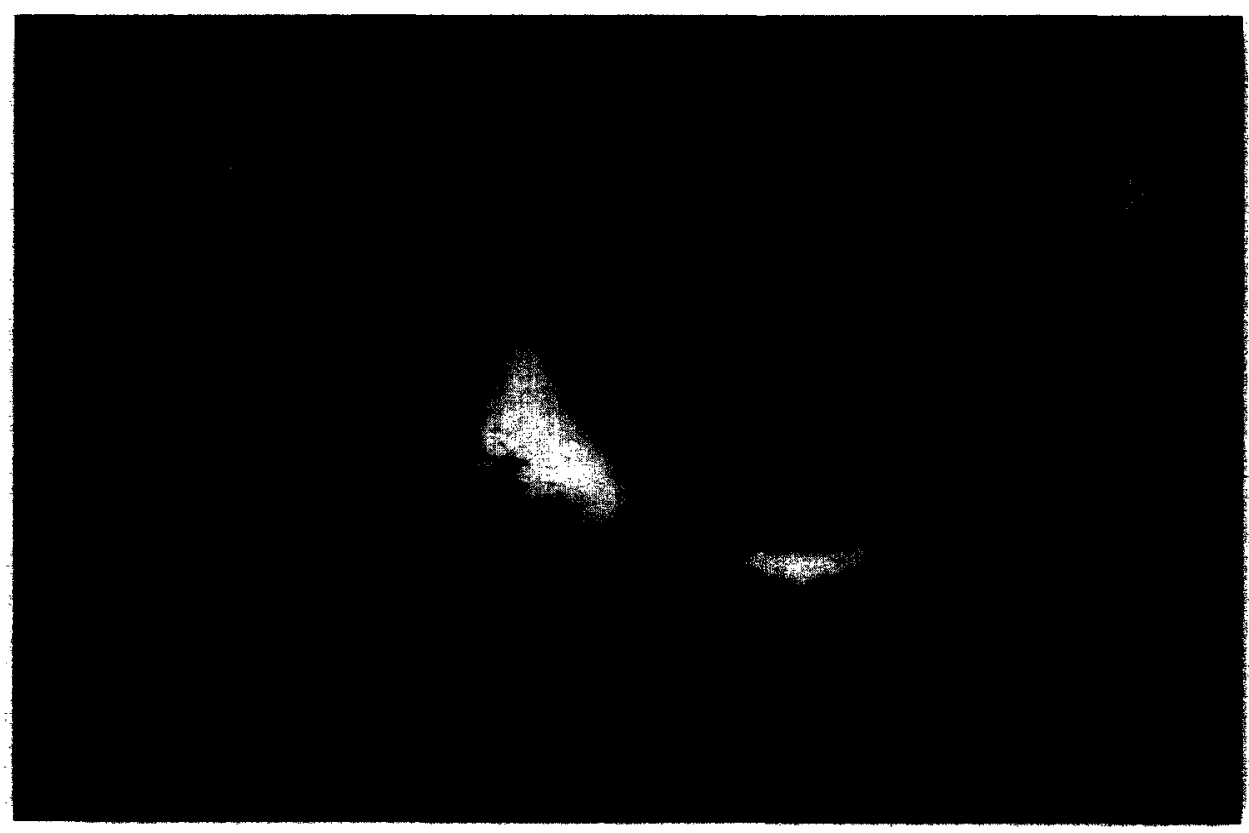

Fig. 6. TEE imaging in a patient with a weight of $1.8 \mathrm{~kg}$, showing a ventricular septal defect. $\mathrm{RA}=$ right atrium; $\mathrm{RV}=$ right ventricle; $\mathrm{LA}=$ left atrium; $\mathrm{LV}=$ left ventricle; $\mathrm{VSD}=$ ventricular septal defect. 


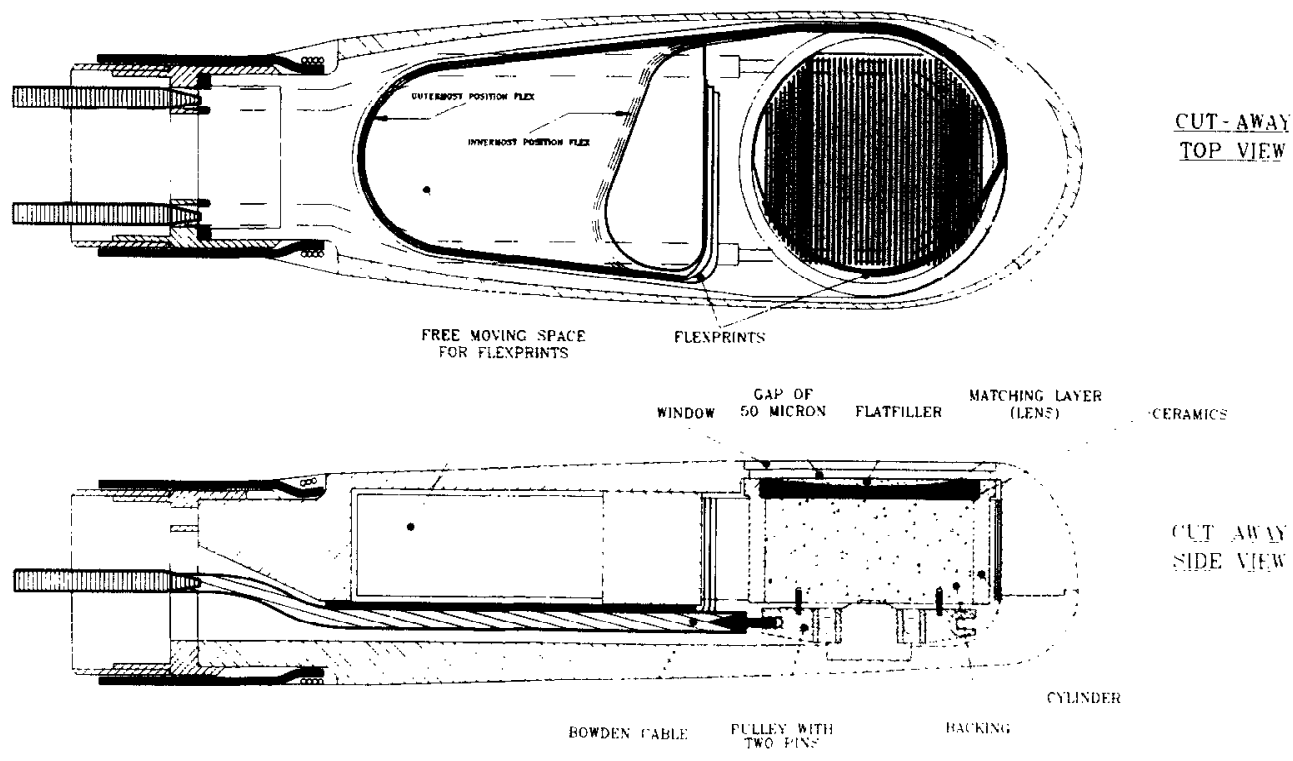

Fig. 7. Schematic cutaway top- and side-view drawing of the pediatric varioplane.

regulations on electrical safety. This prototype probe is not equipped with a thermistor. Acoustic measurements show that the pediatric varioplane has a centre frequency of $5 \mathrm{MHz}$. The $-20-\mathrm{dB}$ bandwidth is $85 \%$ and the round trip insertion loss $7 \mathrm{~dB}$.

At the Sophia Children's Hospital in Rotterdam this probe has been used in over 25 patients (CrommeDijkhuis et al. 1994). Sector as well as Doppler imaging ( CW, PW, color Doppler) are excellent. Clinical experience of the pediatric varioplane demonstrated its value in the ability of visualizing almost every segment of major cardiac structures and especially in visualizing more complex heart disease. Figure 8 shows a subvalvular aortic stenosis imaged with the Varioplane at an imaging plane normally not accessible from mono- or biplane TEE.

The optimal plane is realized with minor probe manipulation and rotational movement of the scanhead leading to better patient comfort. Furthermore, the potential of scanning intermediate planes may allow for a better mentally based three-dimensional (3D) conceptualization of the heart. In the near future, dynamic 3D echocardiography using a pediatric varioplane will also become possible, offering visual three-dimensional conceptualization. This feature is very especially promising in the sometimes complex geometric structures of patients with congenital anomalies of the heart.

\section{FUTURE TEE PROBE DEVELOPMENTS}

TEE probes are valuable expansions of diagnostic tools for assessment of diseases of the heart and great vessels. However, with standard single plane transduc- ers, imaging is limited to the transverse plane. While biplane imaging partially overcomes this lack of versatility of imaging planes, varioplane transducers further expand the diagnostic capabilities since now an infinite number of imaging planes can be obtained while the transducer position in the esophagus is fixed. Still, the above scanning methods are restricted to only $2 \mathrm{D}$ scanning, whereas the heart is a complex 3D organ.

Three-dimensional cardiac imaging based on ultrasound has therefore been in the minds of clinicians for many years. Advances in computer technology combined with developments in ultrasound instrumentation may now lead to dynamic 3D echocardiography in the near future, literally introducing a new dimension to current possibilities of cardiac imaging. In current practice, two methods are being used to obtain 3D ultrasound images.

The first is a real-time method by which a pyramidal burst of ultrasound is generated by a specially designed bow-tie-shaped, matrix transducer. Since this method, developed by von Ramm and colleagues, is in real time, there is no need for spatial and temporal registration. This system, however, exists only as a transthoracic prototype and, to date, the clinical application of this method is still limited (Sheikh et al. 1991).

The second and most common technique is the off-line reconstruction of objects from a consecutive series of 2D images applying imaging formats including linear and rotational scanning (Belohlavek et al. 1993). Since the series of $2 \mathrm{D}$ images have to be matched to each other both in space as well as in time, spatial locator and gating (ECG/respiratory) systems 


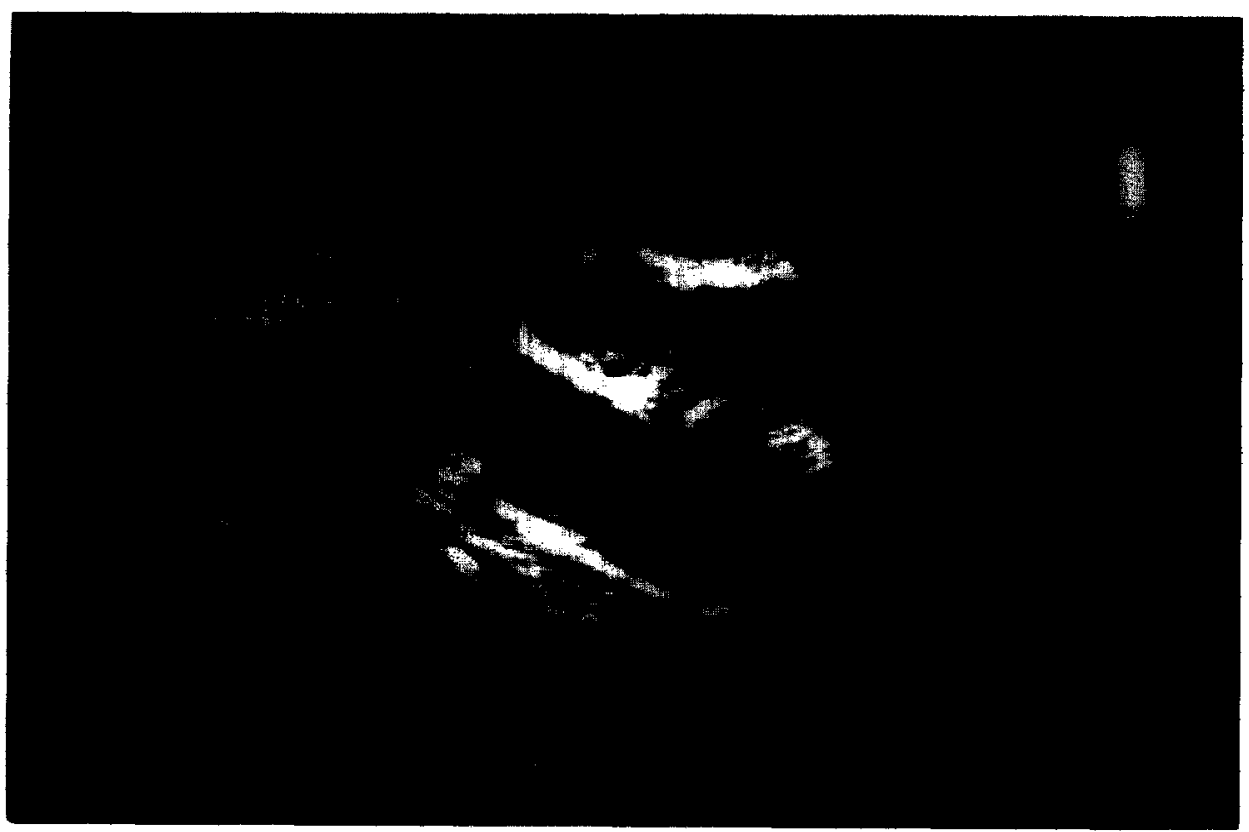

Fig. 8. Varioplane imaging in a patient with a subvalvular aortic stenosis. PA = pulmonary artery; RV = right ventricle; $\mathrm{AO}=$ aorta; $\mathrm{LV}=$ left ventricle. Arrow indicates the subvalvular membrane.

are necessary. On the other hand, the off-line method is applicable to transthoracic and transesophageal probes. Images can be obtained from standard available mechanical annular array as well as electronic phased array TEE probes making it most likely for future TEE probes to be designed for or adapted to the linear (parallel slicing) or rotational (fanlike and propellerlike) 3D acquisition method.

In the parallel slicing method an echo transducer is linearly moved in a flexible tube which has been placed and stretched in the esophagus. This TEE-probe is more suitable for $3 D$ reconstruction rather than for diagnostic use. Beside a large and complicated construction, the probe is actually only a single plane probe. Rotational fanlike and propellerlike scanning methods both have point entry characteristics in which the transducer is first positioned at the esophagus. In the fanlike method, the transducer is then rotated like a fan perpendicular to its imaging plane. Such a probe also can be considered as a single plane probe, while for diagnostic use multiplanes like the varioplane are generally preferred.

After positioning the transducer, varioplanes allow for propellerlike rotational scanning 3D acquisition as well as maximum scanning versatility. As varioplane TEE probes are widely used and have gained clinical acceptance, for TEE the rotational 3D acquisition method using varioplanes seems to be the most promising even if the 3D image quality obtained by each of the above scanning methods would be comparable.

Dynamic cardiac 3D reconstruction is expected to be especially useful in pediatric cardiology where complexly structured congenital heart anomalies visualized in three dimensions may help in better understanding and hence better diagnosis. Our institute is currently working on a small $7.5 \mathrm{-MHz}$ pediatric/neonatal TEE varioplane probe. This probe will have facilities to perform 3D reconstructions with a stepper motor, end switches and an angle rotation indication device. The use of a high centre frequency of $7.5 \mathrm{MHz}$ allows for small dimensions and improved resolution.

\section{CONCLUSIONS}

Transesophageal echocardiography has been a major advance in the semiinvasive assessment of cardiac disease. In the past two decades developments in electronics and (micro) production technology led to smaller TEE probes with increasing image quality. Due to the remarkable size reduction of TEE probes from $18 \mathrm{~mm}$ to less than $10 \mathrm{~mm}$ nowadays, TEE investigations are much better tolerated by the patient and follow-up studies have become possible.

In the beam pattern simulations it was shown that, over the years, considerable beam pattern improvement and, in particular, grating lobe suppression has been obtained. Grating lobes are decreased by more than 20 $\mathrm{dB}$ and the resolution cell by a factor of two or three due to the application of higher frequencies. This evolution of TEE probes to higher frequencies has also led to better visibility of the texture of tissues due to the frequency-dependent scattering. 
Important expansions of the possibilities of TEE scanners were realized by the incorporation of Doppler imaging. Pulsed Doppler, color flow and continuous Doppler only became possible after the development of the 64-clement phased array. The phascd array was divided and care was taken in the design to avoid an overly high-power dissipation.

Another important development was the design of multiplanar devices, first with the biplane and later with the even more versatile varioplane. Rapid advances in dynamic $3 \mathrm{D}$ echocardiography will allow improved diagnostic capabilities in the near future and call for customized transducers. Therefore, future TEE probes should allow for the acquisition of volumetric 3D data as well as optimal diagnostic features. In this respect, varioplanes seem to have the best perspectives, since they offer the possibility for 3D echocardiography as well as maximum scanning versatility.

The availability of smaller coaxial cables, improvements in flexible print technology and application of higher frequencies will lead to further miniaturization of TEE probes and improved image resolution. Especially in pediatric cardiology such small TEE probes are indispensable for diagnosing complex heart defects, for monitoring interventional catheterisation or postoperative evaluation.

Acknowledgements - The authors are grateful for the support and cooperation of Oldelft BV, and for the great amount of work done for our institute at the Central Instrumentation Division of the Erasmus University, in particular by Leo Bekkering.

\section{REFERENCES}

Belohlavek, M.; Foley, D. A.; Gerber, T. C.; Kinter, T. M.: Greenleaf, J. F.; Seward, J. B. Three- and four-dimensional cardiovascular ultrasound imaging: A new era for echocardiography. Mayo Clin. Proc. 68:221-240; 1993.

Bom, N. New concepts in echocardiography. Ph.D. thesis. Leiden: Stenfert Kroese; 1972.

Cromme-Dijkhuis, A. H.; Djoa, K. K.; Bom, N.; Hess, J. Multiplane transesophageal echocardiography in congenital heart defects: An initial experience [abstract]. Circulation 90:I-154, 1994

De Jong, N.; Bom, N.; Lancée, C. T. Esophageal echocardiography. IEEE Trans. Biomed. 8:3-6; 1986.

DiMagno, E. P.; Buxton, J. L.; Regan, P. T.; Hattery, R. R.; Wilson, D. A.: Suarez, J. R.; Green, P. S. Ultrasonic endoscope. Lancet 1:629; 1980

Edler. I.; Hertz, C. H. The use of the ultrasonic reflectoscope for the continuous recordings of the movements of heart walls. $\mathrm{K}$. Fysiogr. Sällsk. Lund. Förh. 24:40-58; 1954.

Frazin, L.; Talano, J. V.; Stephanides, L.; Loeb, H. S.: Kopel, L.: Gunnar, R. M. Esophageal echocardiography. Circulation $54: 102 ; 1976$.

Gussenhoven, E. J.; Taams, M. A.; Roelandt, J. R. T. C.; Bom, N.; Honkoop, J.; De Jong, N.; Ligtvoet, C. M. Esophageal echocardiography. Int. J. Card. Imag. 2:231-239; 1987.

Hanrath, P.: Schlüter, H.; I angenstein, B. A.; Polster, I.; Fngel, S. Transesophageal horizontal and sagittal imaging of the heart with a phased array system. Initial clinical results. In: Hanrath, $\mathbf{P}$., et al., eds. Cardiovascular diagnosis by ultrasound. The Hague: Martinus Nijhoff; 280-288:1982.

Hisanaga, K.; Hisanaga, A.; Nagata, K.; Yoshida, S. A new transesophageal real-time two-dimensional echocardiographic system using a flexible tube and its clinical application. Proc. Jpn. Soc. Ultrason. Med. 32:43-44; 1977.

Hisanaga, K.; Hisanaga, A.; Ichie, Y. A new transesophageal realtime linear scanner and initial clinical results. Proc. Jpn. Soc. Ultrason. Med. 35:115 116; 1978.

Lancée, C. T.; Ligtvoet, C. M.; De Jong, N. On the design and construction of a transesophageal scanner. In: Hanrath, P., et al., eds. Cardiovascular diagnosis by ultrasound. The Hague: Martinus Nijhoff; 1982:260-269.

Lancée, C. T. A transesophageal phased array transducer for ultrasonic imaging of the heart. Ph.D. Thesis, Rotterdam: Erasmus University Rotterdam; 1987.

Lancée, C. T.; de Jong, N.; Bom, N. Design and construction of an esophageal phased array probe. Med. Prog. Technol. 13:139148; 1988.

Omoto, R.; Kyo, S.; Matsumura, M.; Maruyama, M.; Yokote, Y. Future technical prospects in biplane transesophageal echocardiography: Use of adult and pediatric biplane matrix probes. Echocardiography 8:713-720; 1991.

Roelandt, J. R. T. C.; Thomson, I. R.; Vletter, W. B.; Brommersma, P.; Bom, N.; Linker, D. T. Multiplane transesophageal echocardiography: Latest evolution in an imaging revolution. J. Am. Soc. Echocardiogr. 5:361-367; 1992a.

Roelandt, J. R. T. C.; Brommersma, P.; Bom, N.; Vletter, W. B.; Taams, M.; Ten Cate, F. J.: Linker, D. T. Multiplane transesophageal echocardiography with a varioplane transducer system. The Thoraxcentre J. 4:38-43; 1992b.

Sheikh, K. H.; Smith, S. W.; Von Ramm, O.; Kisslo, J. Real-time three-dimensional echocardiography: Feasibility and initial use. Echocardiography 8:119-125; 1991 .

Side, C. G.; Gosling, R. G. Non-surgical assessment of cardiac function. Naturc 232:335; 1971.

Somer, J. C. Electronic sector scanning for ultrasonic diagnosis. Ultrasonics 6:153-159; 1968.

Souquet, J.; Hanrath, P.; Zitelli, L. Transesophageal phased array for imaging the heart. IEEE Trans. Biomed. Eng. 29:707; 1982.

Souquet, J. Phased array transducer technology for transesophageal imaging of the heart: current status and future aspects. In: Hanrath, P., et al., eds. Cardiovascular diagnosis by ultrasound. The Hague: Martinus Nijhoff; 1982:251 259.

Thurstone, F. L.; Von Ramm, O. T. Electronic beam scanning for ultrasonic imaging. In: De Vlieger, M., et al., eds. Ultrasonics in medicine. Amsterdam: Excerpta Medica: 1974:43-48. 